\title{
Pressure tuning of strain in CdTe/InSb epilayer: A photoluminescence and photomodulated reflectivity study
}

\author{
Mark S. Boley, Robert J. Thomas, Meera Chandrasekhar, and H. R. Chandrasekhar \\ Department of Physics and Astronomy, University of Missouri, Columbia, Missouri 65211
}

\author{
A. K. Ramdas \\ Department of Physics. Purdue University. West Lafayette, Indiana 47907 \\ M. Kobayashi and R. L. Gunshor \\ School of Electrical Engineering, Purdue University, West Lafayette, Indiana 47907
}

(Received 31 March 1993; accepted for publication 27 May 1993)

\begin{abstract}
The heavy-hole and light-hole excitons of a CdTe epilayer, pseudomorphically grown on an InSb epilayer by molecular beam epitaxy, are studied with a diamond anvil cell as a function of applied hydrostatic pressure via photoluminescence (PL) and photomodulated reflectivity (PR) spectroscopies. They are compared with the excitonic features in the simultaneously measured PL spectra of a sample of bulk CdTe. Under applied pressure, the lattice mismatch-induced splitting between the light-hole and heavy-hole related transitions increases in a continuous and reversible manner because of the additional pressure-induced compression due to the difference in the compressibilities of CdTe and InSb. The unusually large strain sustained by the CdTe epilayer under pressure is discussed in the light of various models. The PR signal vanishes after the InSb epilayer goes through a structural phase transition at approximately $20 \mathrm{kbar}$, while the PL signal persists until it is irreversibly quenched by the CdTe epilayer undergoing a structural phase transition at approximately $30 \mathrm{kbar}$. For pressures between 20 and $30 \mathrm{kbar}$, the behavior of the CdTe epilayer is similar to that of the bulk sample; the strain appears to have been relaxed due to the structural phase transition which has taken place in InSb. Values of the first- and second-order pressure coefficients for bulk CdTe and for the CdTe epilayer as well as values of the hydrostatic and shear deformation potentials are obtained at 14 and $80 \mathrm{~K}$ and compared with previously quoted values.
\end{abstract}

\section{INTRODUCTION}

High quality epilayers of II-VI semiconductors on epilayers and substrates of III-V materials, grown by molecular beam epitaxy, have aroused much interest in recent years in the context of devices incorporating such unusual strained-layer superlattices. ${ }^{1}$ They offer the possibility of valence band tuning via the interlayer strain. ${ }^{2}$ In view of the lattice mismatch between the II-VI and III-V layers, it is essential to know the conditions of pseudomorphic growth in which the interlayer strain is accommodated by lattice mismatch rather than by the formation of misfit dislocations. The latter are undesirable because of their deleterious effects on the electronic and optical properties of a semiconductor device. As a result of the difference in compressibilities and/or thermal expansion coefficients of the materials forming the heterostructure, the lattice constants of the two constituent materials change in a different manner as a function of pressure and/or temperature, leading to modifications in the stress experienced by the pseudomorphic layer. This leads to a splitting of the otherwise degenerate light- and heavy-hole bands; in a quantum well structure, different well depths for the light and the heavy hole occur. It is therefore of vital importance to know accurately the hydrostatic and uniaxial deformation potentials of the bulk constituents in order to characterize the strain-induced shifts in the valence band as a function of temperature and/or pressure.

In this paper, we present a study of the pressure tuning of the strain experienced by a pseudomorphic CdTe film on an InSb epilayer using (i) photomodulated reflectivity $(P R)$, for observing the heavy- and light-hole excitonic signatures; (ii) photoluminescence (PL), for deducing the relative energies of the heavy-hole excitonic transition at 14 $\mathrm{K}$ with respect to those of the simultaneously measured excitonic transition in a sample of bulk CdTe. The initial biaxial compression due to lattice mismatch increases in magnitude with the application of hydrostatic pressure in the epilayer, leading to a larger splitting between the lightand heavy-hole bands. From these two studies, we have obtained accurate linear and sublinear pressure coefficients for the appropriate transitions. These coefficients, together with the known elastic constants of $\mathrm{CdTe}$ and InSb, have then been used to obtain accurate values of the hydrostatic and uniaxial deformation potentials of CdTe.

\section{EXPERIMENT}

The pseudomorphic epilayer of CdTe under study is $1.3 \mu \mathrm{m}$ thick grown by molecular beam epitaxy (MBE) at a growth temperature of $300^{\circ} \mathrm{C}$ on a (100) surface of an MBE-grown InSb buffer layer on an InSb substrate. The CdTe epilayer was grown in a chamber separate from that in which the InSb buffer layer was grown and the transfer took place in ultra high vacuum. The small lattice mismatch of $0.05 \%$ between the CdTe and the InSb was accommodated by the deformation of the lattice constant of the epilayer while maintaining registry between succes- 
sively grown layers. ${ }^{3}$ For the $\mathrm{CdTe} / \mathrm{InSb}$ system it has been found that coherent pseudomorphic growth is possible up to about $2 \mu \mathrm{m} ;{ }^{4,5}$ above this critical thickness, dislocations are introduced and the lattice constant of the epilayer approaches that of bulk CdTe as revealed in transmission electron microscopic (TEM) studies.

The CdTe/InSb epilayer sample was thinned to $30 \mu \mathrm{m}$ by polishing the substrate, cleaved into a piece about 100 $\times 100 \times 30 \mu \mathrm{m}^{3}$ and loaded cryogenically along with a small cleaved sample of bulk CdTe of similar dimensions into a variable temperature, high-pressure diamond anvil cell of the Merrill-Bassett type, ${ }^{6}$ with argon as the pressure transmitting medium. The pressure was calibrated via the fluorescence from the $R_{1}-R_{2}$ ruby lines emanating from two small chips of ruby also loaded into the cell with the samples.

Photoluminescence was recorded at $14 \mathrm{~K}$ using a closed cycle helium refrigeration system and a $0.85-\mathrm{m}-$ focal-length double-grating Spex model 1401 spectrometer equipped with a thermoelectrically cooled GaAs photomultiplier and photon-counting electronics. The luminescence was excited with the $5145 \AA$ line of an $\mathrm{Ar}^{+}$laser using a power of about $10 \mathrm{~mW}$ incident on a spot size of 50 $\mu \mathrm{m}$ diameter. PL data were obtained in the pressure range of 0-30 kbar, beyond which CdTe undergoes a structural phase transition from the zincblende semiconductor structure to a metallic rocksalt phase with a $20 \%$ change in volume $^{7}$ and the corresponding introduction of a large number of defects, and hence quenches the luminescence irreversibly.

The photomodulated reflectivity (PR) spectra were recorded at $80 \mathrm{~K}$ using a liquid nitrogen continuous flowcryostat and a single-grating Spex model 1702 spectrometer set for a dispersion of $3.2 \AA$ and equipped with a GaAs photomultiplier tube and a lock-in amplifier. A $6 \mathrm{~mW}$ He-Ne laser ( $6328 \AA$ line) was used for photomodulation with powers of about $0.02 \mathrm{~mW}$ over a spot size of $200 \mu \mathrm{m}$ diameter. The modulation frequency used in this experiment was $200 \mathrm{~Hz}$. PR data were obtained in the pressure range of 0-20 kbar, beyond which InSb undergoes a structural phase transition from a zincblende semiconductor structure to a metallic white tin phase, ${ }^{8}$ and the PR signal is irreversibly quenched by a change in volume of the substrate and the corresponding destruction of the pseudomorphic interface between the CdTe epilayer and the InSb epilayer. Both the PL and PR signals were processed and the spectra were plotted with a microcomputer. Details are available elsewhere. ${ }^{9}$

\section{THEORETICAL CONSIDERATIONS}

\section{A. Strains due to lattice mismatch}

The strains in the pseudomorphic CdTe epilayer due to the lattice mismatch are characterized by

$$
\begin{aligned}
& \epsilon=\epsilon_{x x}=\epsilon_{y y}=\frac{a_{s}-a_{e}}{a_{e}}, \quad \epsilon_{z z}=-2 \frac{c_{12}}{c_{11}} \epsilon, \\
& \epsilon_{x y}=\epsilon_{y z}=\epsilon_{z x}=0,
\end{aligned}
$$

where $a_{s}$ and $a_{e}$ are the lattice constants of InSb and CdTe, 6.4741 and $6.4774 \AA$, respectively, at $80 \mathrm{~K}$, and $c_{11}$ and $c_{12}$ are the elastic stiffness constants of CdTe. ${ }^{10}$ Designating the direction of growth of the CdTe epilayer as the $z$ axis, the anisotropic strain is given by the above formula for $\epsilon=\epsilon_{x x}=\epsilon_{y y}$. The main effect of the biaxial compression on the direct $(k=0)$ band of zincblende semiconductors is to shift and split the heavy- and light-hole bands which are normally degenerate in the absence of strain. ${ }^{11,12}$ The hydrostatic $\left(a_{c v}\right)$ and shear $(b)$ deformation potential constants determine the extent of this effect for the compressive stress originating from the lattice mismatch as discussed in Refs. 2 and 3, for example. For an epilayer which has a lattice constant larger than that of the substrate, as is the case in $\mathrm{CdTe} / \mathrm{InSb}$ or in $\mathrm{ZnSe} / \mathrm{GaAs}$, the biaxial stress due to the initial lattice mismatch is compressive in nature and a corresponding band-gap expansion occurs with a heavy-hole-derived band gap.

\section{B. Strains due to applied pressure}

The application of hydrostatic pressure reduces the lattice constants of a material and generates a pressureinduced biaxial stress in the epilayer and the substrate due to the difference in the compressibilities of the two semiconductors. This pressure-induced strain $\epsilon(P)$ can be quantitatively formulated from expressions for the change in the respective lattice constants of the two materials as follows: ${ }^{2,13}$

$$
\epsilon(P)=\left(\frac{a_{s}}{a_{e}}\right)\left[\frac{P}{\left(c_{11}+2 c_{12}\right)_{e}}-\frac{P}{\left(c_{11}+2 c_{12}\right)_{s}}\right],
$$

where $P$ is the applied hydrostatic pressure, and the $c_{i j}$ 's are the elastic stiffness constants for the epilayer $(e)$ and the substrate $(s)$. The net strain experienced by the pseudomorphic layer under the applied pressure is then given by the sum of the initial lattice mismatch strain in Eq. (1) and the pressure-induced strain in Eq. (2). If the bulk modulus of the epilayer is greater than that of the substrate/buffer layer, as is the case in the system under consideration (the $80 \mathrm{~K}$ bulk moduli of $\mathrm{CdTe}$ and InSb are 485.7 and $473.8 \mathrm{kbar},{ }^{10}$ respectively), then the pressureinduced stress is biaxially compressive. Since the initial lattice mismatch also yields biaxial compression, the heavy-light-hole band splitting increases further with the application of hydrostatic pressure. We note that this is the reverse of the situation observed in $\mathrm{ZnSe} / \mathrm{GaAs}^{2,13}$ where the initial lattice mismatch produces compression but the applied pressure causes tension, and a crossover of the heavy- and light-hole bands is observed at a pressure where the two effects exactly cancel each other.

The pressure coefficient of the band gap of the material forming the epilayer in its bulk form is given by

$$
\alpha_{\text {bulk }}=\left[-3 a_{c v} /\left(c_{11}+2 c_{12}\right)\right],
$$




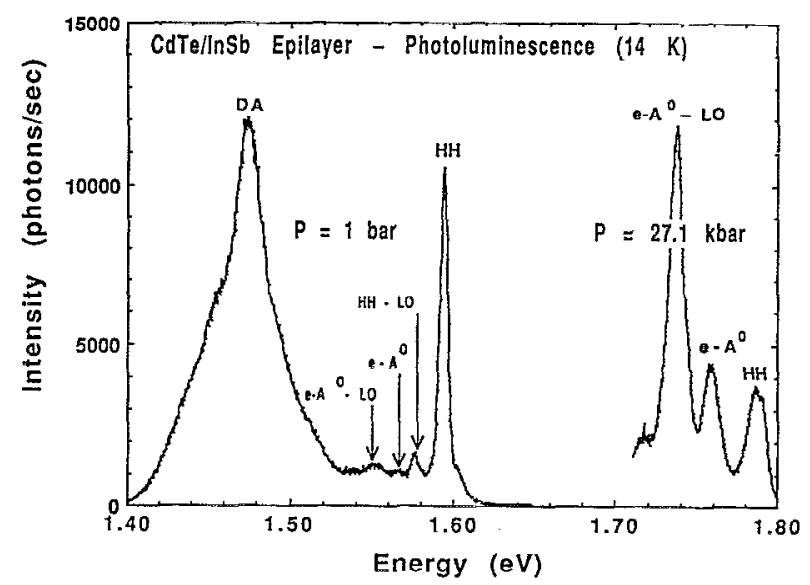

FIG. 1. The photoluminescence spectra for the CdTe/InSb epilayer at 1 bar and $27.1 \mathrm{kbar}$, respectively, both recorded at temperature $T=14 \mathrm{~K}$.

and the pressure coefficients for the light- and heavy-hole band gaps in the strained epilayer can then be determined from the following formula:, 23

$$
\alpha_{\mathrm{hh}}^{\mathrm{lh}}=\alpha_{\mathrm{bulk}}+\left[2 a_{c v} \frac{\left(c_{11}-c_{12}\right)}{c_{11}} \pm b \frac{\left(c_{11}+2 c_{12}\right)}{c_{11}}\right]_{e}\left(\frac{d \epsilon}{d P}\right)
$$

where the $+(-)$ sign in the second term is for the lighthole (heavy-hole) band edges.

\section{RESULTS AND DISCUSSION}

Figure 1 shows the PL spectra for the CdTe/InSb epilayer at $14 \mathrm{~K}$ with no externally applied pressure $(P=1$ bar) and at a pressure near the maximum reached in this experiment $(P=27.1 \mathrm{kbar})$. The PL data were all taken at $14 \mathrm{~K}$, since the PL intensity was found to decrease with increasing temperature. Five prominent transitions can be seen in these spectra. The high-energy peak at $1.595 \mathrm{eV}$ corresponds to the bound exciton associated with the heavy-hole valence-to-conduction-band transition $(\mathrm{HH})$. The pcak at $1.577 \mathrm{eV}$ can be identified as the LO-phonon replica of the heavy-hole transition (HH-LO), since the LO phonon has been determined to have an energy of approximately $18 \mathrm{meV}$ from Raman scattering results. ${ }^{10}$ The peak at $1.565 \mathrm{eV}$ is due to an electron-to-acceptor transition $\left(e-\mathrm{A}^{\mathrm{O}}\right)$, and the peak at $1.550 \mathrm{eV}$ is its LO-phonon replica $\left[\left(e-\mathrm{A}^{0}\right)-\mathrm{LO}\right]$. The lowest energy peak at $1.476 \mathrm{eV}$ has been attributed to donor-acceptor (DA) recombination. ${ }^{14}$ The intensities of the $\mathrm{DA}$ and the $e-\mathrm{A}^{0}$ peaks relative to the $\mathrm{HH}$ peak are found to increase as the externally applied hydrostatic pressure becomes larger, so that the LO-phonon replica of the HH peak can no longer be seen at higher pressures. ${ }^{14}$ The separation between the $e-\mathrm{A}^{0}$ and the $\mathrm{HH}$ peaks decreases slightly with pressure due to the different rate of change of electron and hole effective masses, whereas the separation between the DA and the $\mathrm{HH}$ peaks is found to increase with pressure due to the smaller pressure coefficient of the DA peak. ${ }^{14}$ It should be noted that the exciton associated with the light-hole valence-to-conduction-band transition cannot be seen here, because the population of the light-hole band is much lower than that of the heavy-hole band due to the Boltzmann thermal population factor $[\exp (-\Delta E / k T)]$, approximately equal to 0.16 for the splitting of about $2.2 \mathrm{meV}$ at $14 \mathrm{~K}$. This factor decreases exponentially with pressure, since the splitting increases linearly with pressure, thus making the light-hole-related PL signal too weak to be seen above the background intensity in the spectra.

The observed broadening in the heavy-hole bound exciton peak, as well as the drop in intensity above 20 kbar, can be attributed to the effects of the structural phase change in the InSb at this pressure, ${ }^{8}$ which affects the nature of the interface between the pseudomorphic CdTe film and the InSb epilayer. The disappearance of the PR signal at this pressure also bears out this observation, since the PR spectrum is highly dependent upon the quality of the surface of the epilayer and the interface between the epilayer and the substrate. This broadening and reduction in intensity in the PL spectra can also be partly attributed to the precursor of the structural phase transition in CdTe at $30 \mathrm{kbar}$, since a similar effect, although of a smaller magnitude, can be seen in the simultaneously measured PL spectra of our sample of bulk CdTe (not shown here) as the externally applied pressure approaches $30 \mathrm{kbar}$. The PL spectra of the bulk CdTe sample also exhibit the bound-exciton peak, the electron-acceptor peak, and the DA peak, as reported in Ref. 14. The heavy- and light-hole bound excitonic transitions are degenerate in energy in the bulk sample.

Figure 2 shows the excitonic transition energies from the PL data obtained as a function of pressure, for the heavy-hole bound exciton peak of the CdTe epilayer, and the degenerate light- and heavy-hole bound exciton peak of the CdTe bulk sample. It should be noted that a point by point comparison can be made between the epilayer and bulk transition energies, without any concern for the uncertainty in pressure measurements between the two samples or due to two different loadings, since the two samples were loaded into the same pressure cell and were therefore at identical pressures at all of the energy versus pressure measurements. The bulk transition energies are found to be most accurately fit by a nonlinear [second order: $\left.E(P)=E(0)+\alpha P+\beta P^{2}\right]$ rather than a linear function of pressure [first order: $E(P)=E(0)+\alpha P$ ], where $P$ is the pressure in kbar. The residual sum of squares was about eight times lower for the nonlinear than for the linear function of pressure. This significant nonlinearity of the bulk transition with pressure can be seen in the bowing of the curve and has been previously observed in other experiments. ${ }^{14}$ The values of $\alpha$ and $\beta$ obtained from the fit, $7.85 \pm 0.12$ and $0.029 \pm 0.004 \mathrm{meV} / \mathrm{kbar}^{2}$ respectively, are also in excellent agreement with our previous results ( $\mathrm{Ta}$ ble I) ${ }^{14}$ The heavy-hole-related transition energies of the CdTe epilayer are found to fit most accurately to a linear function of pressure with $\alpha=7.17 \pm 0.06 \mathrm{meV} / \mathrm{kbar}$. The fit was limited to the $0-20 \mathrm{kbar}$ range due to the onset of the phase transition in the InSb substrate.

In the absence of externally applied pressure, the exci- 


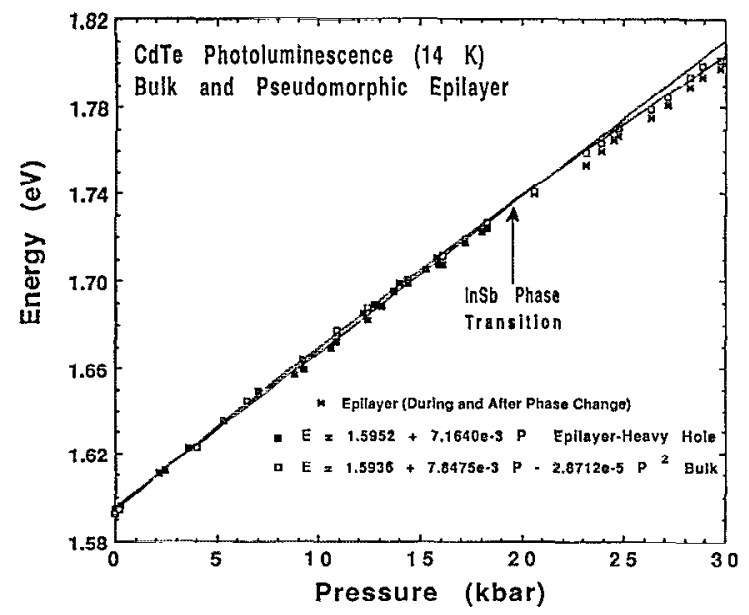

FIG. 2. The CdTe/InSb epilayer heavy-hole transition energies and the CdTe bulk transition energies vs pressure from the PL data at $14 \mathrm{~K}$, fit with first- and second-order polynomials in pressure, respectively. Note that the epilayer heavy-hole transition energies for pressures beyond 20 kbar (denoted with a different symbol) follow closely the bulk transition energies rather than the straight-line fit, due to the phase transition that has taken place in the InSb substrate.

tonic transition energy in the bulk CdTe lies below that of the CdTe epilayer heavy-hole exciton, but at a pressure of approximately 2 kbar they coincide. As can be seen in Fig. 2 , the bulk transition energy then lies above that of the CdTe epilayer heavy-hole transition up to approximately $20 \mathrm{kbar}$ where they again coincide. Beyond $20 \mathrm{kbar}$, the bulk transition energy again lies below the extrapolation of the linear fit from 0 to $20 \mathrm{kbar}$ for the epilayer heavy-hole transition energies, but the actual epilayer data between 20 and $30 \mathrm{kbar}$ are also seen to deviate from this extrapolation of the 0 to $20 \mathrm{kbar}$ linear fit. The actual epilayer heavy-hole transition energies continue to lie just below the bulk transition energies by almost a constant amount of $\approx 5 \mathrm{meV}$ in the pressure range 20 to $30 \mathrm{kbar}$ and the shape of the curve for the epilayer transition energies in this range is the same as that of the bulk curve with the corresponding bowing as the pressure is increased. However, this bowing was not present in the epilayer below $20 \mathrm{kbar}$ and can therefore be attributed to the effect produced in the epilayer of $\mathrm{CdTe}$ by the structural phase transition in InSb. It appears that the strain in the CdTe epilayer has relaxed above $20 \mathrm{kbar}$ since the epilayer transition energies become bulk-like in their pressure characteristics. This is most likely due to the destruction of the heterointerface after the structural phase transition in InSb; the CdTe epilayer then becomes essentially free-standing. The PL signal from both the CdTe epilayer and the bulk sample irreversibly vanish at pressures above $30 \mathrm{kbar}$ due to the structural phase transition in the Cd'le from the zincblende structure into the nonluminescent metallic rocksalt phase.

Figure 3 shows the PR spectra for the CdTe/InSb epilayer at $80 \mathrm{~K}$ and $P=1$ bar in panel (a), and at a pressure near to which the PR signal vanished $(P=17.5 \mathrm{kbar})$ in panel (b). All the PR spectra were taken at $80 \mathrm{~K}$, because the best signal to noise ratio was achieved at that temperature; at lower temperatures, the PR signal is difficult to distinguish above the luminescence background. Three transitions are present in these spectra, as can be clearly determined by a three oscillator fit to the PR data (also shown in the figure) with the functional form ${ }^{15}$

$$
\frac{\Delta R}{R}(E)=\sum_{j=1}^{3} \operatorname{Re}\left[C_{j} e^{i 0_{j}}\left(E^{\prime}-E_{j}+i \Gamma_{j}\right)^{-n}\right]
$$

where $C_{j}$ and $\theta_{j}$ are the amplitude and asymmetry of the line shape, and $E_{j}$ and $\Gamma_{j}$ are the energy and width of the transitions, respectively. The $n$ value used was two, characteristic of two-dimensional excitonic line shapes. ${ }^{16}$ This expression is convenient for numerical fits and yields line positions and widths that are in excellent agreement with other functions available in the literature. Also shown in Fig. 3 are the "pseudoabsorption" spectra" at these two pressures, constructed from a sum of three Lorentzians with their energy positions, widths, and integrated intensi-

TABLE I. First- and second-order pressure coefficients and hydrostatic/shear deformation potentials for CdTe from the data of Figs. 2 and 4.

\begin{tabular}{|c|c|c|c|c|c|c|}
\hline Sample & Transition & $\begin{array}{c}\text { Energy at } P=0 \\
(\mathrm{eV})\end{array}$ & $\begin{array}{c}\alpha \\
\text { (meV/kbar) }\end{array}$ & $\begin{array}{c}\beta \\
\left(\mathrm{meV} / \mathrm{kbar}^{2}\right)\end{array}$ & $\begin{array}{c}a_{c v} \\
(\mathrm{eV})\end{array}$ & $\begin{array}{c}b \\
(\mathrm{eV})\end{array}$ \\
\hline $\mathrm{CdTe}$ (bulk) & degenerate & 1.5936 & 7.85 & -0.029 & -3.81 & -0.76 \\
\hline $\mathrm{PL}$ at $14 \mathrm{~K}$ & $\mathrm{HH}$ and $\mathrm{LH}$ & \pm 0.0008 & \pm 0.12 & \pm 0.004 & \pm 0.06 & \pm 0.24 \\
\hline $\mathrm{CdTe}$ (epilayer) & HH & 1.5953 & 7.17 & $\cdots$ & $\cdots$ & -0.76 \\
\hline $\mathrm{PL}$ at $14 \mathrm{~K}$ & & \pm 0.0007 & \pm 0.06 & & & \pm 0.24 \\
\hline $\mathrm{CdTe}$ (epilayer) & $\mathrm{HH}$ & 1.5858 & 7.43 & $\cdots$ & -3.71 & -0.91 \\
\hline $\mathrm{PR}$ at $80 \mathrm{~K}$ & & \pm 0.0019 & \pm 0.19 & & \pm 0.09 & \pm 0.18 \\
\hline $\mathrm{CdTe}$ (epilayer) & LH & 1.5880 & 7.94 & $\cdots$ & -3.71 & -0.91 \\
\hline $\mathrm{PR}$ at $80 \mathrm{~K}$ & & \pm 0.0021 & \pm 0.20 & & \pm 0.09 & \pm 0.18 \\
\hline \multirow{2}{*}{\multicolumn{3}{|c|}{ Previous measurement (bulk CdTe) }} & $7.59^{a}$ & $-0.029^{a}$ & $-3.4^{\mathrm{b}}$ & $-1.4^{\mathrm{b}}$ \\
\hline & & & \pm 0.19 & \pm 0.007 & \pm 0.6 & \pm 0.4 \\
\hline \multicolumn{3}{|c|}{ Previous theoretical calculation (bulk CdTe) } & $5.5^{c}$ & $\cdots$ & $-2.72^{r}$ & $\cdots$ \\
\hline
\end{tabular}

${ }^{2}$ Reference 14.

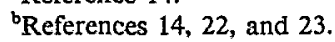

'Reference 21 . 

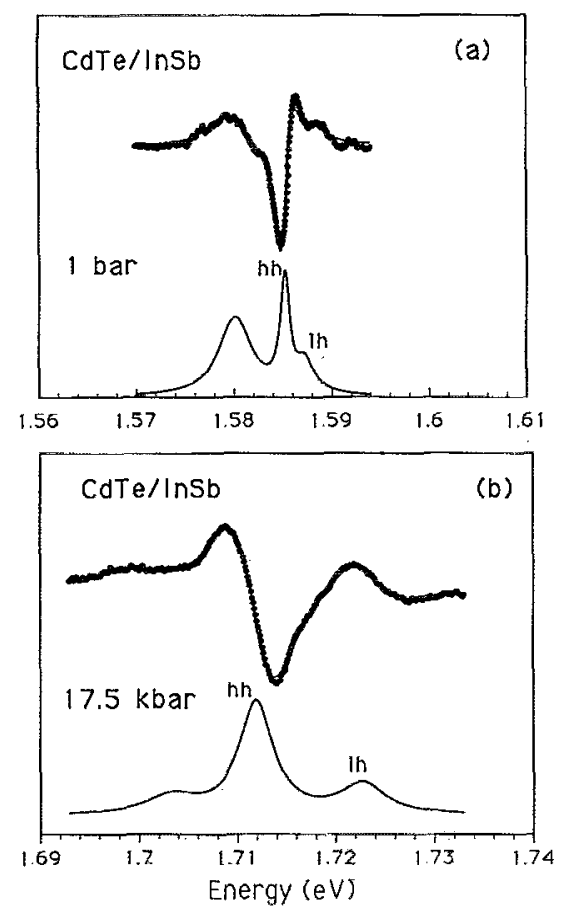

FIG. 3. The photomodulated reflectivity and "pseudoabsorption" spectra for the $\mathrm{CdTe} / \mathrm{InSb}$ epilayer at (a) $1 \mathrm{bar}$ and (b) at $17.5 \mathrm{kbar}$, respectively, and at $80 \mathrm{~K}$.

ties being calculated from the fitted parameters. The higher energy, lower intensity transition at $1.587 \mathrm{eV}$ is attributed to the light-hole ( $\mathrm{LH})$ valence to conduction-band transition, ${ }^{3}$ while the lower energy, higher intensity transition at $1.585 \mathrm{eV}$ is attributed to the heavy-hole ( $\mathrm{HH})$ valence-to-conduction-band transition. ${ }^{4}$ The relative intensities are as expected from a comparison of the optical matrix elements for these transitions, ${ }^{17}$ and their relative energies are consistent with the compressive stress in the sample. The third transition, at a still lower energy of 1.580 $\mathrm{eV}$ and of much lower intensity, is believed to be associated with an exciton bound to a mechanical-damage-induced defect, either a line defect such as a dislocation or a pointdefect-impurity complex, which becomes more intense after the mechanical thinning process. ${ }^{3,18-20}$ The light- and heavy-hole transitions move up in energy to 1.723 and $1.712 \mathrm{eV}$, respectively, at $17.5 \mathrm{kbar}$, and the separation between them increases as the pressure is increased. The PR signal vanishes above $20 \mathrm{kbar}$, due to the deleterious effects of the structural phase transition of the InSb epilayer on the surface of the CdTe epilayer and on the initially pseudomorphic interface between them.

Figure 4 shows the CdTe epilayer excitonic transition energies from the PR data obtained as a function of pressure. Both the heavy- and light-hole transitions are accurately fit by a linear function of the pressure of the form $E(P)=E(0)+\alpha P$ where $P$ is the pressure in kbar, with pressure coefficients of $7.43 \pm 0.19$ and $7.94 \pm 0.20 \mathrm{meV} /$ kbar, respectively, for heavy- and light-hole-related transitions (Table I). In the absence of externally applied pressurc, the light-hole transition lies above the heavy-hole

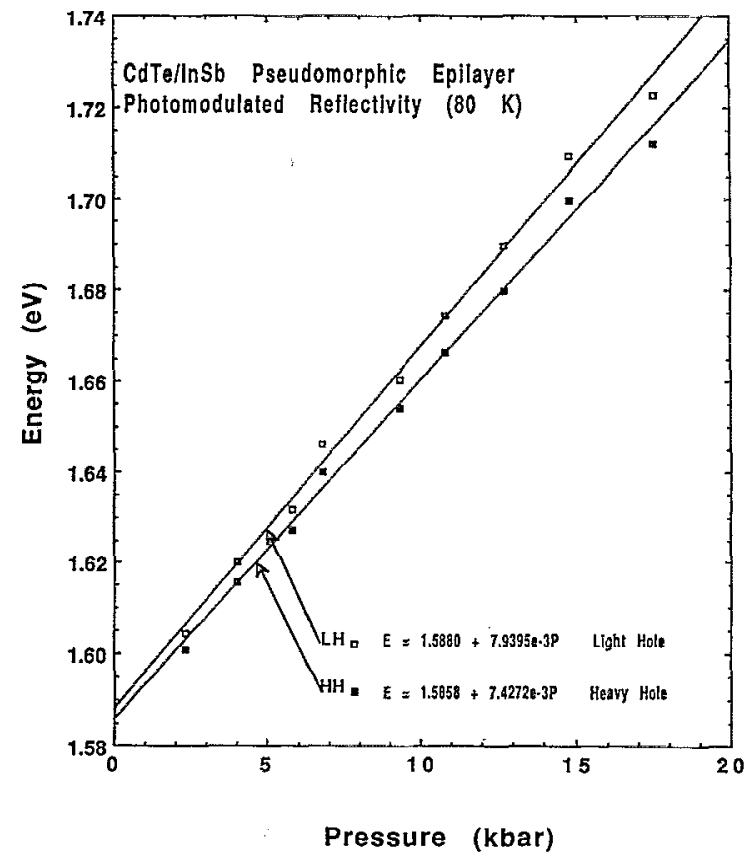

FIG. 4. The CdTe/InSb epilayer heavy- and light-hole transition energies vs pressure from the PR data at $80 \mathrm{~K}$, fit with first-order polynomials in pressure. Note that the heavy-light-hole energy separation increases linearly with pressure.

transition in energy, in agreement with the compressive nature of the initial lattice mismatch strain in the CdTe epilayer. As the pressure is increased, the light- and heavyhole transitions separate further in energy, as the pressureinduced compressive stress adds to the initial compressive stress. The heavy-hole/light-hole splitting is directly dependent upon the amount of strain present in the epilayer, and thus increases linearly with pressure from approximately $2 \mathrm{meV}$ at 1 bar to approximately $13 \mathrm{meV}$ at $20 \mathrm{kbar}$, as can be seen more clearly in Fig. 5, where we plot the heavy/light-hole energy separation in $\mathrm{meV}$ as a function of pressure, and fit the data to a straight line. It should be noted that the energy at a pressure of 1 bar, $E(P=0)$, obtained from the linear fit of the heavy-hole transition energies of the PR data is in excellent agreement with $E(P$ $=0)$ obtained from the fit to the epilayer heavy-hole transition energies of the PL data, when the energy correction for the temperature shift from 80 to $14 \mathrm{~K}$ is included ( $\approx 10$ $\mathrm{meV}$ ). In addition, it should also be noted that the linear pressure coefficient $\alpha$ obtained from the fit to the PR data for the $\mathrm{HH}$ at $80 \mathrm{~K}$ is almost equal within uncertainties to that obtained from the fit to the PL data for it at $14 \mathrm{~K}$.

We calculate the hydrostatic deformation potential $a_{c v}$ for CdTe in two ways, using either PL or PR data. Using Eq. (3) with the linear pressure coefficient $\alpha$ for the bulk material (CdTe) obtained from the fit to our PL data, we can calculate $a_{c v}$ at $14 \mathrm{~K}$, using the known values of the elastic stiffness constants for CdTe: $c_{11}=607.1 \mathrm{kbar}$, $c_{12}=424.5 \mathrm{kbar}$ and for InSb: $c_{11}=679.7 \mathrm{kbar}, c_{12}=372.2$ kbar at 14 and $80 \mathrm{~K} .{ }^{10}$ Using this method we obtain the hydrostatic deformation potential $a_{c v}$ at $14 \mathrm{~K}$ for $\mathrm{CdTe}$ to be equal to $-3.81 \pm 0.06 \mathrm{eV}$. On the other hand, as sug- 


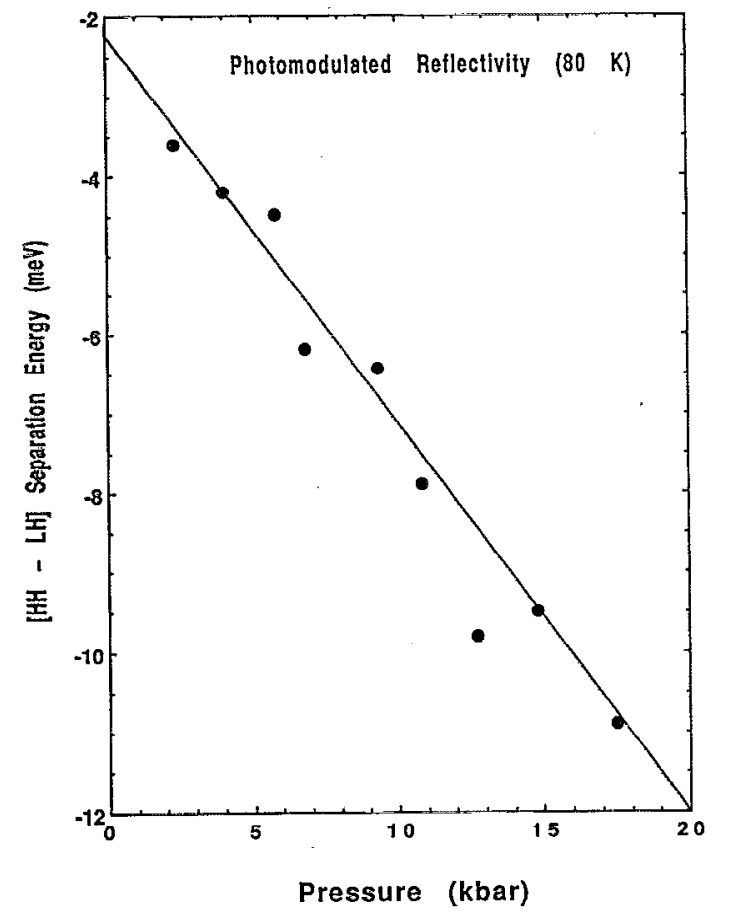

FIG. 5. The CdTe/InSb epilayer heavy-hole (HH)-light-hole (LH) energy separation in $\mathrm{meV}$ vs pressure from the PR data at $80 \mathrm{~K}$. The separation increases linearly with pressure by a factor of approximately 6 in the pressure range $0-20 \mathrm{kbar}$.

gested by Eq. (4) we can compute $a_{c v}$ at $80 \mathrm{~K}$ from the average of the light- and heavy-hole linear pressure coefficients ( $\alpha$ 's) obtained from the fits to our PR data for CdTe to be equal to $-3.71 \pm 0.09 \mathrm{eV}$. These values can be compared with a calculated value of $a_{c v}$ from Cardona and Christensen of $-2.72 \mathrm{eV},{ }^{21}$ and an experimental value of $-3.4 \pm 0.6 \mathrm{eV}$ from uniaxial stress measurements in CdTe by Thomas, ${ }^{22}$ but corrected with the new bulk pressure coefficient $\alpha$ obtained from the second-order fit to our PL data and also quoted by Prakash et al., ${ }^{14}$ instead of the previously used bulk pressure coefficient $\alpha$ obtained from a first-order fit to PL data. ${ }^{23}$ The two values of $a_{c v}$ which we have obtained are in agreement with each other within their uncertainty limits, and clearly demonstrate that $a_{c v}$ does not change significantly with temperature between 14 and $80 \mathrm{~K}$.

We can also calculate the shear deformation potential $b$ for CdTe from the PL data and the PR data. Using the 1 bar pressure splitting between the epilayer heavy-hole transition and the bulk excitonic transition $\left[E^{\text {hh }}(P=0)-E^{\text {bulk }}(P=0)\right]$ and the linear pressure coefficient for the bulk material obtained from the fitted PL data at $14 \mathrm{~K}$, and the compressive initial lattice mismatch strain in the epilayer calculated as in Eq. (1) above, as well as the known values of the elastic stiffness constants for the epilayer material ( $\mathrm{CdTe}$ ), we can calculate the value of the shear deformation potential $b$ at $14 \mathrm{~K}$ from the following equation:

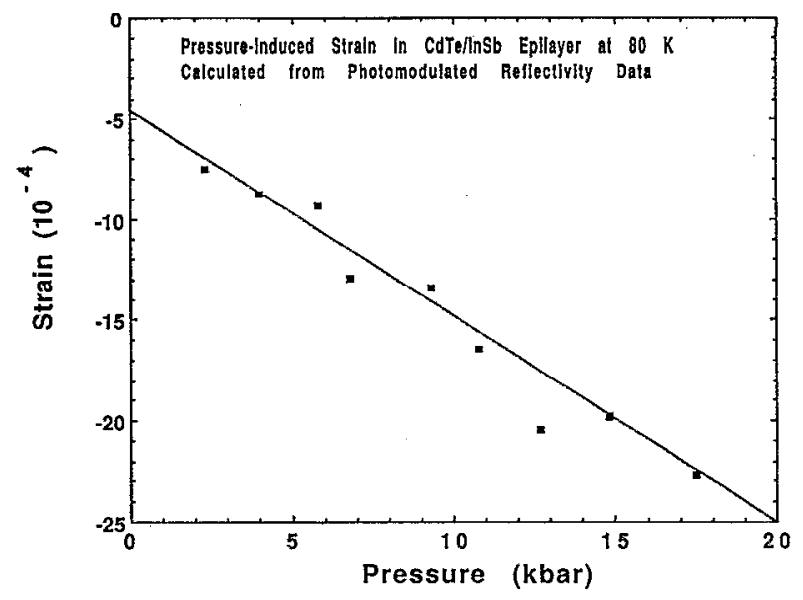

FIG. 6. The pressure-induced strain in the CdTe epilayer arising from the differences in the compressibilities of CdTe and InSb over the range 0-20 kbar. The strain was calculated using the heavy-hole/light-hole splitting from the PR data at $80 \mathrm{~K}$ and an average value of the shear deformation potential $b$.

$$
\begin{aligned}
E_{g}^{\mathrm{hh}}(P & =0)-E_{g}^{\mathrm{bulk}}(P=0) \\
& =-\left(\frac{c_{11}+2 c_{12}}{c_{11}}\right)_{e}\left[\frac{2}{3} \alpha_{\mathrm{bulk}}\left(c_{11}-c_{12}\right)_{e}+b\right] \epsilon(P=0)
\end{aligned}
$$

and we obtain $b=-0.76 \pm 0.24 \mathrm{eV}$ for $\mathrm{CdTe}$ at $14 \mathrm{~K}$ in this manner. An independent value for $b$ can be obtained from the 1 bar pressure splitting between the epilayer heavy-hole and light-hole transitions $\left[E^{\mathrm{hh}}(P=0)\right.$ $\left.-E^{\mathrm{hh}}(P=0)\right]$ deduced from the fitted PR data at $80 \mathrm{~K}$, along with the compressive initial lattice mismatch strain and the elastic stiffness constants of CdTe. The shear deformation potential $b$ at $80 \mathrm{~K}$ is calculated from the following equation:

$$
E_{g}^{\mathrm{hh}}(P=0)-E_{g}^{\mathrm{hh}}(P=0)=-2 b\left(\frac{c_{11}+2 c_{12}}{c_{11}}\right)_{e} \epsilon(P=0),
$$

which yields $b=-0.91 \pm 0.18 \mathrm{eV}$ for CdTe at $80 \mathrm{~K}$. This can be compared to the previous value of $b$ obtained from uniaxial stress measurement by Thomas, ${ }^{22}$ viz. $-1.4 \pm 0.4$ $\mathrm{eV}$, after correction with our bulk linear pressurc coefficient $\alpha{ }^{14,23}$ The two values of $b$ thus obtained are in agreement with each other within their uncertainty limits and demonstrate that $b$ does not significantly vary with temperature between 14 and $80 \mathrm{~K}$.

Using an average value for the shear deformation potential $b$ and the heavy-hole/light-hole splittings from the PR data displayed in Fig. 5, we can calculate the pressureinduced strain $\epsilon(P)$ in the epilayer of CdTe, shown in Fig. 6 , where a straight line fit has been made to the data. As can be seen from this figure, the absolute magnitude of the strain increases by approximately a factor of 6 as the pressure increases from 1 bar to $20 \mathrm{kbar}$. At pressures higher than $20 \mathrm{kbar}$ the strain in the CdTe epilayer relaxes and it begins to behave like the bulk material. It should be noted 
that the large value of the pressure-induced strain just below $20 \mathrm{kbar}$ where it begins to relax is far greater than the initial lattice mismatch strain allowed under the conditions prescribed for pseudomorphic growth. Yet the sample interface retains its pseudomorphic character and the strain in the epilayer continues to grow linearly with pressure as predicted by theory even beyond the pseudomorphic limit. This can perhaps be explained by the large amount of pressure to which the sample is subjected and may be connected with some previously observed "superpressing" effects. $^{24}$

The unexpectedly large strain sustained by the epilayer at high pressures raises certain questions, since the layer is fairly thick $(1.3 \mu \mathrm{m})$. The accommodation of lattice mismatch through strain has been discussed by several authors. ${ }^{25}$ For growth of an epilayer on a $\langle 100\rangle$ substrate, edge dislocations with a Burgers vector of $b=\frac{1}{2} a[110]$ are found to be the predominant mechanism of producing misfit dislocations. Minimizing the energies due to the elastic strain and the energy of an edge dislocation at a distance $h$ from the substrate, ${ }^{25}$ one obtains the largest possible value of strain, $\epsilon^{*}$ between two crystal surfaces under equilibrium conditions,

$$
\epsilon^{*}=\frac{b}{8 \pi h(1+v)}\left[\ln \left(\frac{h}{b}\right)+1\right],
$$

where $b=\frac{1}{2} a[110]=(1 / \sqrt{2}) a=4.6 \AA$ in magnitude for $\mathrm{CdTe}$ and $v$ is Poisson's ratio $=-s_{11} / s_{12}=0.4$ for $\langle 100\rangle$ growth, where $s_{11}$ and $s_{12}$ are the elastic compliance constants. In principle, the largest strain sustainable would be for a thickness of one monolayer, $h_{m}$. For CdTe, this value would be $h_{m}=a / 2$, giving $\epsilon^{*}=2.8 \%$. This corresponds to a maximum stress of $\epsilon^{*} / s_{11}=7.3 \mathrm{kbar}$ since $1 / \mathrm{s}_{11}=261 \mathrm{kbar}$ (Ref. 10) is Young's modulus for this stress direction. In practice, bulk CdTe breaks ${ }^{22}$ at about 2 kbar due to slippage of the planes, and the real maximum strain should be closer to $0.8 \%$. We find that the maximum strain that we have attained is about a quarter of this value (Fig. 6). It may have been possible to reach a higher strain if InSb had not transformed to a different structure; however, experiments on a different combination of materials will be needed to test these limits. The large shear sustained, does, however raise the question of how far the pseudomorphic limit can be exceeded, especially at these temperatures, which are well below those at which the sample is grown.

We present three ways of looking at the problem in order to relate the magnitude of interfacial strain achieved by pressurc to quantities used in understanding the growth of pseudomorphic layers and the conditions under which misfit dislocations are produced; one way is to calculate the equilibrium critical thickness for the known shear strain using Eq. (8); the second is to calculate the areal energy density due to the strain; and the third is to calculate the excess strain that drives the dislocations.

Figure 7(a) shows a plot of the expected critical thickness $h_{c}$, the maximum thickness an epilayer can have without misfit dislocations for a given lattice mismatch. The value of $h_{c}$ is given by Eq. (8), substituting $h_{c}$ for $h$ and using the strain for a given epilayer-substrate combination
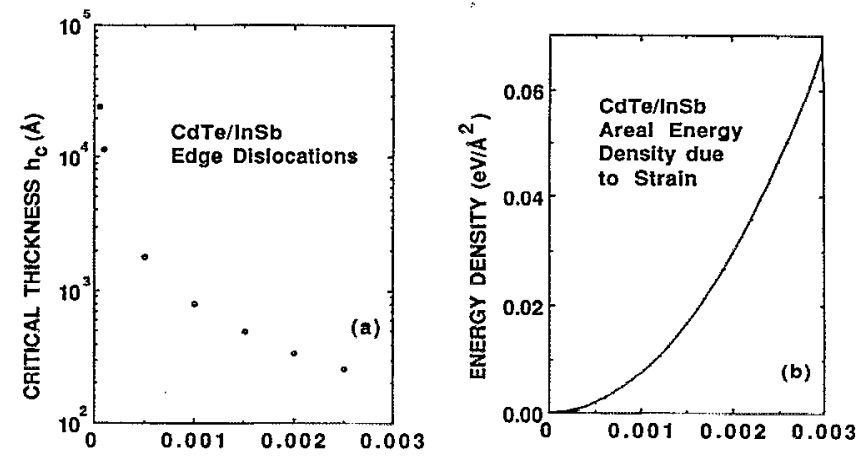

LATTICE MISPATCH

LATTICE MISMATCH STRAIN
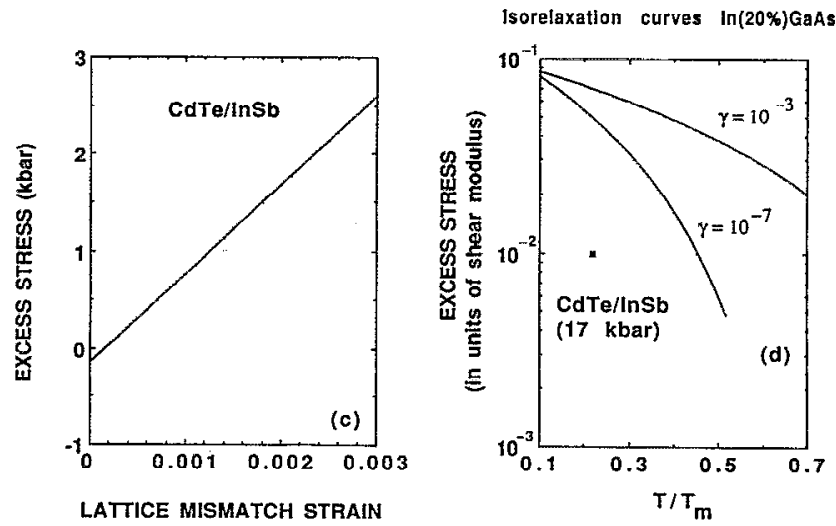

FIG. 7. (a) Calculated values of critical thickness $h_{c}$ for the range of strains shown in Fig. 6. (b) The areal energy density for a 1.3- $\mu$ m-thick layer for the range of strains shown in Fig. 6, calculated using Eq. (9). (c) The excess stress that drives dislocation motion for the strains in Fig. 6, calculated using Eq. (10). (d) Isorelaxation curves for $\mathrm{In}_{0.2} \mathrm{Ga}_{0.8} \mathrm{As} / \mathrm{GaAs}$ extended to the temperature range $T / T_{m}$ relevant to our experiment. The largest excess stress achieved in our experiment is indicated and is below the level of the $\gamma=10^{-7}$ curve which indicates the pscudomorphic limit in $\operatorname{In}_{0,2} \mathrm{Ga}_{0.8} \mathrm{As} / \mathrm{GaAs}$.

for $\epsilon^{*}$. At atmospheric pressure, for the $\mathrm{CdTe} / \mathrm{InSb}$ combination, this value is close to our layer thickness of 1.3 $\mu \mathrm{m}$. When we tune the strain with pressure (Fig. 6), the theoretical value of $h_{c}$ should change with pressure. This expected change is shown in Fig. 7(a), for the range of $\epsilon$ spanned in Fig. 6, and calculating $h_{c}(\epsilon)$ using Eq. (8). For the highest strain generated $(\epsilon=0.0023$ at $17 \mathrm{kbar})$, the critical thickness should be only about $300 \AA$. Correspondingly, the areal energy density ${ }^{25}$ given by

$$
E_{\varepsilon}=\frac{2 G(1+v)}{(1-v)} \epsilon^{2} h,
$$

where $h$ is the layer thickness $(1.3 \mu \mathrm{m})$, and $G$ is the shear modulus, $C_{44}=196 \mathrm{kbar}$ for $\langle 100\rangle$ strain. We see from Fig. 7 (b) that the energy density increases by a factor of 20 between ambient pressure and $17 \mathrm{kbar}$. Apparently the strain energy provided is still inadequate to relax the layer through the production of a large number of misfit dislocations, since we still have a measurable strain and no apparent discontinuity in $\epsilon$.

It is, of course, not possible to determine from our measurements whether or not a small fraction of the strain 
is relieved, and what we observe is just the residual strain. When dislocations are produced, one expects that there will be inhomogeneities in the strain across different domains, while our experiment measures only the average strain. We do observe an increase in the FWHM linewidth of the HH transition from about 1.75 to about $4 \mathrm{meV}$. If this is indicative of the shear strain inhomogeneity, $|\Delta \epsilon|$, then $|\Delta \epsilon|<2 \times 10^{-4}$ at the maximum strain of $\epsilon=23 \times 10^{-4}$. An independent measure of the degree of relaxation via the measurement of the interface lattice constant $a_{\|}$is necessary to determine the dislocation density.

In the extreme limit where the pressure-induced interfacial strain creates dislocations, it should be possible to determine the activation energy for creating dislocations. To our knowledge, the activation energy for dislocation glide has been determined in few semiconductor systems, among them $\mathrm{SiGe} / \mathrm{Si}(1.1 \pm 0.2 \mathrm{eV})$ (Ref. 26) and InGaAs/GaAs (1.2 eV) (Ref. 27), via the tuning of the lattice mismatch with alloying. In order to compare how close our sample is to strain relief, we calculate the excess stress in the epilayer, and attempt to compare it to the excess stress required, at room temperature, to produce plastic flow.

The excess stress that drives dislocation motion and hence strain relief in the epilayer is given by ${ }^{28}$

$$
\sigma_{\mathrm{ex}}=\frac{2 G(1+v)}{(1-v)}|\epsilon|-\frac{G\left(1-v \cos ^{2} \beta\right)}{2 \pi(1-v)} \frac{\ln (4 h / b)}{h / b},
$$

where $\beta$ describes the orientation of the dislocation, usually found to be at $60^{\circ}$ to the Burgers vectors. ${ }^{25}$ The excess stress for our epilayer is calculated using the strain in Fig. 6 and Eq. (10), and is shown in Fig. 7(c). We note that the excess stress increases from $0.3 \mathrm{kbar}$ at ambient pressure to about 2 kbar at $17 \mathrm{kbar}$.

Scaling relations have been developed ${ }^{29}$ that allow a prediction of the amount of strain relaxation where plastic deformation is dominated by glide mechanisms. Based on a single measurement of relaxation on a particular system of materials, the dependence of strain relaxation on lattice mismatch, layer thickness, and temperature can be predicted. The result of this phenomenological model is that stability diagrams of isorelaxation curves can be drawn via plots of excess stress (in units of $G$ ) versus growth temperature (in units of melting temperature $T_{m}$ ). The relaxation is defined as the fractional difference in the in-plane lattice parameter ${ }^{28}$

$$
\gamma=\left(a_{\|}-a_{s}\right) / a_{s},
$$

where $a_{\|}$is the epilayer lattice constant parallel to the interface and $a_{s}$ is the substrate lattice constant. In a pseudomorphic film, the in-plane lattice parameter $a_{\|}$ matches that of the underlying substrate and $\gamma$ is very small, while $\gamma$ is significantly larger for a relaxed layer. It is found in Ref. 29 that for SiGe and InGaAs systems that a $\gamma$ value of $10^{-7}$ indicates close to pseudomorphic structure, whilc $10^{-3}$ indicates a relaxed structure. To our knowledge, the required single measurement is not available for CdTe. However, a comparison of the curves for SiGe and InGaAs/GaAs gives an indication of the direction of the curves expected for a II-VI material. Extending the curves shown in Ref. 27 for the InGaAs/GaAs system to the 300 $K$ range [Fig. 7 (d)], we see that the pseudomorphic limit corresponds to an excess stress of $\sigma_{\text {ex }} / G=0.046$ at $300 \mathrm{~K}$ $\left(T / T_{m}=0.235\right)$. In contrast, in our CdTe epilayer, $\sigma_{\mathrm{ex}} / G=0.01$, a factor of 4 smaller. It is possible that the pseudomorphic isorelaxation curves of the softer II-VI material are pushed down to lower excess stresses, as they are in InGaAs as compared to that in SiGe. It therefore appears that at $17 \mathrm{kbar}$ our sample is fairly close, but probably below the excess stress required to produce dislocations at $300 \mathrm{~K}$.

\section{CONCLUSIONS}

The present work demonstrates how externally applied hydrostatic pressure can be used to characterize the built-in strains in the constituents of a semiconductor heterostructure. It has been clearly shown that the techniques of PL and PR spectroscopies can be used independently to study the strain in a heterostructure, and that they give results which are in good agreement. The stress in an epilayer of CdTe, pseudomorphically grown on an epilayer of InSb, initially compressive due to the small lattice mismatch between the two materials could be made, with the application of external hydrostatic pressure, more compressive in a reversible manner below the threshold of the structural phase transitions which occur in the InSb substrate and the CdTe epilayer at approximately 20 and 30 kbar, respectively. Accurate values of the hydrostatic and shear deformation-potential constants and their temperature dependence have emerged from this study using PL and PR spectroscopies, and comparison with the PL spectra of a simultaneously measured sample of bulk CdTe. Furthermore, $\mathrm{ZnTe} / \mathrm{AlSb} / \mathrm{GaSb}^{30}$ and $\mathrm{ZnTe} / \mathrm{InAs}$ epilayers have been fabricated with MBE by us, ${ }^{31}$ and these materials are currently under consideration in order to explore analogous effects.

\section{ACKNOWLEDGMENTS}

The work by H.R.C. was supported in part by the U.S. Department of Energy under Contract No. DE-FG0289ER45402. M.C. acknowledges partial support from the Research Corporation and the U.S. Army Grant No. DAAL-03-92-G-0381. M.S.B. acknowledges partial support by the G. Ellsworth Huggins Fellowship. A.K.R. and R.L.G. acknowledge support from the National Science Foundation (Materials Research Group No. DMR8913706). We thank Lok C. Lew Yan Voon, L. R. RamMohan, and N. Otsuka for many stimulating discussions.

${ }^{1}$ R. L. Gunshor, L. A. Kolodziejski, A. V. Nurmikko, and N. Otsuka, in Strained Layer Superlattices: Materials Science and Technology, edited by T. P. Pearsall, Semiconductors and Semimetals, edited by R. K. Willardson and A. C. Beere (Academic, New York, 1991), Vol. 33, pp. 337-409.

${ }^{2}$ B. Rockwell, H. R. Chandrasekhar, M. Chandrasekhar, A. K. Ramdas, M. Kobayashi, and R. L. Gunshor, Phys. Rev. B 44, 307 (1991).

${ }^{3}$ Y. R. Lee, A. K. Ramdas, L. A. Kolodziejski, and R. L. Gunshor, Phys. Rev. B 38, 143 (1988).

${ }^{4}$ R. L. Gunshor, L. A. Kolodziejski, M. R. Melloch, M. Vaziri, C. Choi, 
and N. Otsuka, Appl. Phys. Lett. 50, 200 (1987); T. Yao, Y. Okada, S. Matsui, K. Ishida, and I. Fujimoto, J. Cryst. Growth 81, 518 (1987).

${ }^{5}$ H. Mitsuhashi, I. Mitsuishi, M. Mizuta, and H. Kukimoto, Ipn. J. Appl. Phys. 24, L578 (1985); K. Ohkawa, T. Mitsuyu, and O. Yamazaki, Phys. Rev. B 38, 465 (1988).

${ }^{6}$ L. Merrill and W. A. Bassett, Rev. Sci. Instrum. 45, 290 (1975).

${ }^{7}$ S. B. Qadri, E. F. Skelton, A. W. Webb, E. R. Carpenter, M. W. Schaefer, and J. K. Furdyna, Phys. Rev. B 35, 6868 (1987).

${ }^{8}$ M. D. Banus and M. C. Lavine, J. Appl. Phys. 40, 409 (1969); S.-C. Yu, I. L. Spain, and E. F. Skelton, J. Appl. Phys. 49, 4741 (1978).

${ }^{9}$ A. Jayaraman, Rev. Mod. Phys. 55, 65 (1983); U. Venkateswaran, M. Chandrasekhar, H. R. Chandrasekhar, B. A. Vojak, F. A. Chambers, and J. M. Meese, Phys. Rev. B 33, 8416 (1986); U. Venkateswaran, Ph.D. thesis, University of Missouri-Columbia, 1985; Y. M. Gupta and Z. A. Shen, Appl. Phys. Lett. 58, 583 (1991).

${ }^{10}$ For material parameters see, for example, S. Adachi, J. Appl. Phys. 58, RI (1985); 53, 8775 (1982), and rcferences therein; Landolt-Bornstein Numerical Data and Functional Relationships in Science and Technology, New Series, Group III, edited by O. Madelung, M. Schultz, and H. Weiss (Springer, Berlin, 1982), Vols. 17a and 17b.

${ }^{11}$ F. H. Pollak, Strained-Layer Superlattices: Physics, edited by T. P. Pearsall (Academic, Boston, 1990).

${ }^{12}$ C. G. Van de Walle, Phys. Rev. B 39, 1871 (1989); E. P. O'Reilly, Semicond. Sci. Technol. 4, 121 (1989).

${ }^{13}$ J. A. Tuchman and I. P. Herman, Phys. Rev. B 45, 11929 (1992); J. A. Tuchman, S. Kim, Z. Sui, and I. P. Herman, ibid. 46, 13371 (1992).

${ }^{14}$ Maneesha Prakash, Meera Chandrasekhar, H. R. Chandrasekhar, I. Miotkowski, and A. K. Ramdas, Phys. Rev. B 42, 3586 (1990).

${ }^{15}$ D. E. Aspnes, Surf. Sci. 37, 418 (1973).

${ }^{16}$ O. J. Glembocki and B. V. Shanabrook, Superlatt. Microstruct. 3, 235 (1987); F. H. Pollak and O. J. Glembocki, Proceedings of the Society of
Photo-Optical Instrumentation Engineers (SPIE, Bellingham, 1988), Vol. 946, p. 2.

${ }^{17}$ F. H. Pollak and M. Cardona, Phys. Rev. 172, 816 (1968).

${ }^{18}$ C. E. Barnes and K. Zanio, J. Appl. Phys. 46, 3959 (1975).

${ }^{19}$ D. J. Leopold, J. M. Ballingall, and M. L. Wroge, Appl. Phys, Lett. 49, 1473 (1986).

${ }^{20}$ Z. C. Feng, M. G. Burke, and W. J. Choyke, Appl. Phys. Lett. 53, 128 (1988).

${ }^{21}$ M. Cardona and N. E. Christensen, Phys. Rev. B 35, 6182 (1987).

${ }^{22}$ D. G. Thomas, J. Appl. Phys. 32, 4310 (1961).

${ }^{23}$ D. J. Dunstan, B. Gil, and K. P. Homewood, Phys. Rev. B 38, 7862 (1988).

${ }^{24}$ B. A. Weinstein, S. K. Hark, R. D. Burnham, and R. M. Martin, Phys. Rev. Lett. 58, 781 (1986); L. J. Cui, U. D. Venkateswaran, B. A. Weinstein, B. T. Jonker, and F. A. Chambers, in Frontiers in High Pressure Research, edited by H. D. Hochheimer and R. D. Etters (Plenum, New York, 1991), pp. 269-280.

${ }^{25}$ J. W. Matthews and A. E. Blakeslee, J. Cryst. Growth, 27, 118 (1974); J. W. Matthews, J. Vac. Sci. Technol. 12, 126 (1975), and references therein.

${ }^{26}$ R. Hull, J. C. Bean, D. J. Werder, and R. E. Leibenguth, Appl. Phys. Lett. 52, 1605 (1988).

${ }^{27}$ P. L. Gourley, I. J. Fritz, and I. R. Dawson, Appl. Phys. Lett. 52, 377 (1988); A George and J. Rabier, Rev. Phys. Appl. 22, 941 (1987).

${ }^{28}$ B. W. Dodson and J. Y. Tsao, Appl. Phys. Lett. 53, 2498 (1988), and references therein.

${ }^{29}$ B. W. Dodson and J. Y. Tsao, Appl. Phys. Lett. 55, 1345 (1989), and references therein.

${ }^{30}$ D. L. Mathine, S. M. Durbin, R. L. Gunshor, M. Kobayashi, D. Menke, Z. Pei, J. Gonsalves, N. Otsuka, Q. Fu, M. Haggerott, and A. V. Nurmikko, Appl. Phys. Lett. 55, 268 (1989).

${ }^{31}$ R. L. Gunshor (unpublished). 